Artículos Científicos

\title{
Los exámenes cooperativos en la educación superior: un enfoque desde la perspectiva del estudiante
}

\begin{abstract}
Cooperative Exams in Higher Education: An Approach from the Student's Perspective
\end{abstract}

Exames cooperativos no ensino superior: uma abordagem da perspectiva do aluno

Alan Ramírez-Noriega Universidad Autónoma de Sinaloa, Facultad de Ingeniería Mochis, México Universidad Autónoma Indígena de México, México alandramireznoriega@uas.edu.mx https://orcid.org/0000-0002-8634-9988

Samantha Jiménez

Instituto Tecnológico de Tijuana, Departamento de Sistemas y Computación, México samantha.jimenez@tectijuana.edu.mx https://orcid.org/0000-0003-0938-7291

Yobani Martínez-Ramírez Universidad Autónoma de Sinaloa, Facultad de Ingeniería Mochis, México yobani@uas.edu.mx https://orcid.org/0000-0002-4967-9187

José Emilio Sánchez García Universidad Autónoma Indígena de México, México esanchez@uaim.edu.mx https://orcid.org/0000-0001-8018-8426 


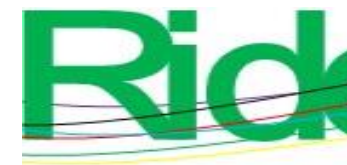

\section{Revista Iberoamericana para la Investigación y el Desarrollo Educativo ISSN 2007 - 7467}

\section{Resumen}

Los exámenes cooperativos impulsan al estudiante a trabajar en pequeños grupos y así, grupalmente, cumplir una meta. Con este tipo de exámenes, los alumnos reciben comentarios inmediatos de sus compañeros mientras contestan el examen. Esta investigación determinó el impacto y la aceptación de los exámenes cooperativos (dos integrantes) desde la perspectiva de estudiantes de ingeniería de Software de la Universidad Autónoma de Sinaloa. La muestra para el experimento se conformó de seis grupos en etapa terminal de dicha carrera profesional. El experimento consistió en resolver exámenes cooperativos a lo largo del semestre. Para recopilar información, una vez finalizado el semestre, los estudiantes contestaron un instrumento previamente diseñado. Los resultados indican una aceptación de los exámenes cooperativos. Además, según los participantes, estos exámenes brindan retroalimentación y reducen el estrés que provoca el ser evaluados.

Palabras clave: educación superior, evaluación, examen cooperativo, trabajo en equipo.

\section{Abstract}

Cooperative exams allow the student to work in small groups and thus, as a group, meet a goal. With these types of exams, students receive immediate feedback from their peers while they answer the exam. This research determined the impact and acceptance of cooperative exams (two members) from the perspective of Software Engineering students from the Universidad Autónoma de Sinaloa. The sample for the experiment was six groups in the terminal stage of said professional career. The experiment consisted of solving cooperative test throughout the semester. To gather information, an instrument was designed and applied with students at the end of the semester. Results indicate an acceptance of the cooperative exams. Besides, according to students, these exams provide feedback and reduce the stress of being evaluated.

Keywords: higher education, evaluation, cooperative exam, teamwork. 


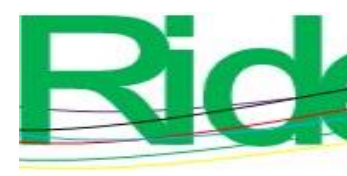

Revista Iberoamericana para la Investigación y el Desarrollo Educativo ISSN 2007 - 7467

\section{Resumo}

Os exames cooperativos incentivam o aluno a trabalhar em pequenos grupos e, assim, como um grupo, atingir um objetivo. Com esses tipos de exames, os alunos recebem feedback imediato de seus colegas à medida que respondem ao exame. Esta pesquisa determinou o impacto e a aceitação dos exames cooperativos (dois membros) da perspectiva dos alunos de engenharia de software da Universidade Autônoma de Sinaloa. A amostra do experimento foi composta por seis grupos em estágio terminal da referida carreira profissional. O experimento consistiu na resolução de exames cooperativos ao longo do semestre. Para a coleta de informações, após o término do semestre, os alunos responderam a um instrumento previamente elaborado. Os resultados indicam uma aceitação dos exames cooperativos. Além disso, de acordo com os participantes, esses testes fornecem feedback e reduzem o estresse de ser testado.

Palavras-chave: ensino superior, avaliação, exame cooperativo, trabalho em equipe. Fecha Recepción: Febrero 2020 Fecha Aceptación: Agosto 2020

\section{Introducción}

La educación es considerada de suma importancia en la sociedad moderna. Sin duda se trata de un área compleja por la imbricación de múltiples factores sociales, económicos y culturales, entre otros. Como bien sabemos, en el núcleo de la educación se encuentra el proceso de enseñanza-aprendizaje. Al respecto, el término enseñanza es definido como la estructuración de las diferentes situaciones educativas de manera que ayuden a los alumnos a cambiar a través del aprendizaje (Johnson y Johnson, 2004). Por otra parte, el aprendizaje es el cambio que se produce en un estudiante por una enseñanza (Johnson y Johnson, 2004).

Para determinar el impacto de la enseñanza en el aprendizaje, se requiere del empleo de procedimientos evaluativos. La evaluación implica reunir información sobre la calidad y la cantidad del cambio experimentado por un alumno o un grupo de alumnos (Johnson y Johnson, 2004); es decir, implica valorar el conocimiento a través de la emisión de un juicio.

La evaluación puede tener muchas funciones. Si bien dar una calificación es la única en muchos docentes, podría usarse para motivar al alumno, reforzar el trabajo hecho y destacar fortalezas y habilidades. Según Díaz y Hernández (2010), existen técnicas e instrumentos para la correcta evaluación del alumno, tales como las técnicas informales, semiinformales y 


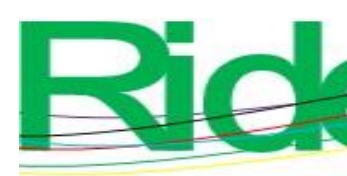

Revista Iberoamericana para la
Investigación y el Desarrollo Educativo
ISSN $2007-7467$

formales. Esta investigación aborda las técnicas formales de evaluación, en específico los exámenes escritos para evaluar teoría y exámenes prácticos. Estas técnicas exigen un proceso de planeación y elaboración más sofisticado y suelen aplicarse en situaciones que demandan un mayor control.

Un examen es un instrumento diseñado para medir el conocimiento y la comprensión del contenido definido. La evaluación es una actividad importante para el alumno, ya que este necesita identificar cómo se desempeñó en el examen y si los resultados le permiten progresar en sus estudios. La evaluación también es importante para el educador. La evaluación es un medio para identificar si la enseñanza es efectiva y qué tan bien el alumno comprende el material (Cantwell, Sousou, Jadotte, Pierce y Akioyamen, 2017).

Una percepción errónea muy habitual es que la enseñanza y la evaluación son actividades independientes. De hecho, la enseñanza mejora considerablemente cuando se integra la evaluación (Johnson y Johnson, 2004). La mayoría de las instituciones educativas evalúan el aprendizaje de los estudiantes con pruebas independientes, es decir, los estudiantes completan la prueba por su cuenta sin la ayuda de compañeros o recursos externos. Una alternativa a este formato tradicional es el examen cooperativo. En esta modalidad cooperativa los alumnos trabajan juntos en pequeños grupos para responder las preguntas del examen (Centrella, 2012; Gilley y Clarkston, 2014).

El aprendizaje cooperativo es un conjunto de métodos, técnicas y estrategias de enseñanza que permiten hacer más participativo y dinámico el proceso de enseñanzaaprendizaje (Alarcón, Sepúlveda y Madrid, 2018; Centrella, 2012; Revelo, Collazos y Jiménez, 2018). La cooperación permite al estudiante compartir perspectivas, debatir puntos, preguntar y entender otros puntos de vista, resolver problemas complejos y alcanzar un acuerdo, entre otros aspectos importantes (Amores, 2016; Mahoney y Harris, 2019). Este tipo de aprendizaje ofrece considerables ventajas al instructor: le facilita la organización de clase y la consecución de los objetivos académicos personales y sociales, le proporciona un conocimiento más completo de sus alumnos y le permite incidir en sus procesos de integración.

El examen cooperativo es una estrategia fundada en el constructivismo social, específicamente dentro de la teoría de la interdependencia social. Según esta perspectiva, los individuos son agentes activos en un proceso continuo de aprendizaje que ocurre como resultado de las interacciones con los demás y el medio ambiente (Díaz y Hernández, 2010; Mahoney y Harris, 2019). El hombre es un ser social por naturaleza: nace, crece y se desarrolla 


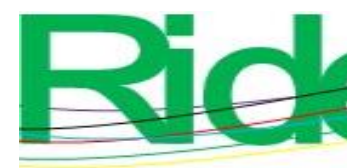

Revista Iberoamericana para la Investigación y el Desarrollo Educativo

ISSN $2007-7467$

en grupo y, aunque el aprendizaje es un proceso individual o independiente, está condicionado socialmente por los grupos de pertenencia. Es por eso por lo que es necesario integrar este tipo de aprendizajes en el ambiente escolar, debido a que de manera natural los estudiantes están hechos para enseñar a otros alumnos, a relacionarse y participar con sus iguales (Alarcón et al., 2018).

Siguiendo la filosofía del aprendizaje cooperativo, esta investigación determina el impacto que tienen los exámenes cooperativos en estudiantes de ingeniería de software. Para lograr esto, se realizó una investigación que diseñó un instrumento para recabar información y conocer la experiencia de alumnos que aplicaron exámenes cooperativos a lo largo de un semestre, si bien ya habían tenido experiencias previas. Por tanto, se buscan respuestas a las siguientes preguntas:

1) ¿Los estudiantes de los semestres finales de la carrera de ingeniería de Software de la Facultad de Ingeniería Mochis de la Universidad Autónoma de Sinaloa aceptan los exámenes cooperativos en pareja?

2) ¿Cuáles ventajas y desventajas perciben los estudiantes al aplicar exámenes cooperativos en pareja?

Este artículo está organizado de la siguiente manera. La segunda sección explica la metodología de trabajo para recopilar la información a través de un experimento. La tercera sección muestra los resultados del experimento y un análisis de los mismos. Para finalizar, se describen las conclusiones y las referencias empleadas en el presente documento.

\section{Metodología}

Los métodos de investigación mixta son una poderosa herramienta de descripción, comprensión y explicación de fenómenos educativos (Núñez, 2017). Estos métodos implican la recolección y el análisis de datos cuantitativos y cualitativos, con el objetivo de profundizar en la problemática desde dos aspectos (Hernández, Fernández y Baptista, 2014).

Esta investigación aplica el método mixto para describir los aspectos relacionados con los exámenes cooperativos y con los estudiantes desde un enfoque cualitativo, además de interpretar datos estructurados y estadísticos con el fin de encontrar relaciones entre variables desde el enfoque cuantitativo. Con esta combinación de métodos se busca determinar la aceptación de los exámenes cooperativos en pareja, así como determinar si hay relaciones entre variables. Los procedimientos de recuperación y análisis de datos incluyen la observación y 


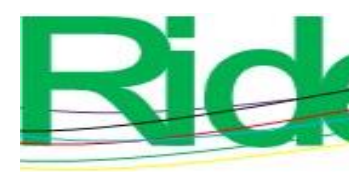

Revista Iberoamericana para la Investigación y el Desarrollo Educativo ISSN $2007-7467$

aplicación de cuestionarios en línea.

\section{Muestra y participantes}

El tipo de muestra es no probabilística por conveniencia. Este enfoque permite seleccionar aquellos casos accesibles que acepten ser incluidos. Esto fundamentado en la conveniente accesibilidad y proximidad de los sujetos para el investigador. Sin embargo, cabe aclarar que los sujetos deben de cumplir con ciertas características afines a los objetivos del experimento (Hernández et al., 2014).

Las clases y las evaluaciones se aplicaron en la Facultad de Ingeniería Mochis de la Universidad Autónoma de Sinaloa. Los estudiantes eran de séptimo y noveno semestre de la carrera de ingeniería de Software. Hubo tres grupos de cada semestre para dar un total de seis grupos. El total de encuestados fue de 55 de 69 estudiantes pertenecientes a los grupos. Algunos no contestaron por diversas razones: no aplicaron exámenes cooperativos en pareja, no se enteraron de la encuesta, desertaron de la carrera o simplemente por no tener disposición a contestar el instrumento.

\section{Procedimiento}

La forma de desarrollar el experimento es la siguiente:

- Se impartieron clases con una evaluación basada en exámenes cooperativos en pareja durante un semestre. Las materias fueron "Sistemas de soporte a la decisión” y "Minería de datos". Fueron tres evaluaciones (una por parcial) por materia y por grupo.

- Se diseñó una encuesta empleando Google Forms ${ }^{1}$. La encuesta contiene seis preguntas de datos generales y nueve preguntas referentes a la experiencia de los estudiantes al aplicar exámenes en pareja.

- La encuesta fue aplicada al terminar el semestre que cursaban los estudiantes en ese momento. Es decir, el primer semestre del ciclo escolar 2019-2020.

- La encuesta se distribuyó a través de Internet. Se envió la encuesta a los jefes de grupo y ellos se encargaron de distribuirla entre sus compañeros.

- Los datos fueron recopilados automáticamente por el sistema de encuestas empleado; incluso el sistema generó algunas gráficas de fácil interpretación.

\footnotetext{
${ }^{1}$ https://www.google.com/intl/es_mx/forms/about/.
} 

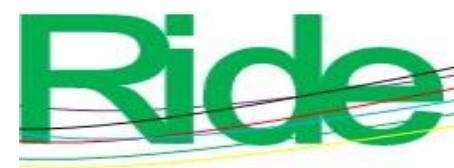

Revista Iberoamericana para la Investigación y el Desarrollo Educativo ISSN 2007 - 7467

- $\quad$ Finalmente, los datos fueron obtenidos y procesados para el análisis.

\section{Encuesta}

La encuesta está dividida en dos aspectos: datos generales y la experiencia con los exámenes. A continuación, se muestran los datos generales recabados por los estudiantes (entre paréntesis el tipo de respuesta a los datos solicitados):

- $\quad$ Sexo (Mujer, Hombre, Prefiero no decirlo)

- $\quad$ Edad (Entero)

- $\quad$ Semestre $(1,2,3,4 \ldots)$

- $\quad$ Promedio actual (Real)

- $\quad$ Grupo $(101,102,103,201,202$, 203, 301, 302, 303, 401, 402, 403, 501, 502,

503)

- ¿Eres alumno regular? (Sí, No)

Estos datos sirven para hacer comparativos entre los distintos atributos recopilados. No se solicitó el nombre del estudiante ni ningún tipo de rastreo para que contestaran con la mayor fiabilidad posible. Respecto a la última pregunta, los alumnos regulares son aquellos que no tienen materias pendientes por aprobar.

Las preguntas donde los encuestados plasmaban su opinión de acuerdo con su experiencia aplicando exámenes cooperativos en pareja son:

- ¿Cuántos exámenes en pareja has contestado aproximadamente en la universidad? (Cuantitativo discreto)

- $\quad$ Me he sentido cómodo aplicando exámenes en pareja (Likert de cinco puntos)

- $\quad$ Considero que los exámenes en pareja refuerzan el conocimiento que tengo débil (Likert de cinco puntos)

- $\quad$ Existe una retroalimentación con mis compañeros al resolver el examen en pareja (Likert de cinco puntos)

- $\quad$ Siento que mis conocimientos son mejores al terminar el examen en pareja que antes de aplicar el examen en pareja (Likert de cinco puntos)

- Los exámenes deberían ser en pareja (Likert de cinco puntos)

- $\quad$ Los exámenes en pareja son útiles en: (Teoría, Práctica, Ambos, Ninguno)

- $\quad$ ¿Cuáles desventajas aprecias al hacer un examen en pareja? (Abierta) 


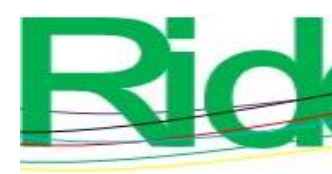

Revista Iberoamericana para la Investigación y el Desarrollo Educativo ISSN 2007 - 7467

- ¿Cuáles ventajas aprecias al hacer un examen en pareja? (Abierta)

\section{Análisis estadístico}

Para el tratamiento y análisis de los datos se emplea la herramienta estadísticas SPSS. ${ }^{2}$ Esta herramienta ofrece un análisis estadístico avanzado, además de que es fácil de usar, flexible y escalable. Asimismo, esta herramienta trae integrada una variedad de gráficas para representar la información de trabajo, lo que permite interpretar los datos de mejor forma. Las gráficas de la investigación y algunas tablas fueron generadas con SPSS.

Con el objetivo de profundizar en la información recabada, se aplicó la prueba estadística de la ji al cuadrado. Para poder aplicar esta prueba se tuvo que aplicar la técnica de estaninos (Thorndike, 1982) en las preguntas de la encuesta donde se empleó la escala de Likert. La técnica consiste en transformar el conjunto de datos agregando dos atributos. El primero representa la sumatoria de las preguntas donde se empleó Likert. El segundo atributo consiste en la discretización del primer atributo. La discretización es generada con dos puntos de cortes considerando la desviación estándar y siguiendo las siguientes fórmulas:

$$
\begin{aligned}
& a=\bar{X}-(0.75 * \sigma) \\
& b=\bar{X}+(0.75 * \sigma)
\end{aligned}
$$

Se generan dos cortes para obtener tres categorías. La categoría uno corresponde a los valores menores al valor del corte $a$ (fórmula 1). La categoría dos corresponde a los valores mayores o iguales al valor del corte $a$ y menores al valor del corte $b$ (fórmula 2). La categoría tres corresponde a los valores mayores o iguales al valor del corte $b$. No se consideró llamar a las categorías como malo, neutral y bueno, como si fuera una escala de Likert, dado que los resultados muestran que no hay opiniones negativas, más bien es una escala globalizada de todas las respuestas empleando Likert.

Se utilizaron las categorías para realizar un análisis intrasujetos, es decir, se aplicó el mismo experimento a todos los estudiantes y se analizaron aspectos internos de la muestra para explorar si los resultados obtenidos tienen relación con algunos aspectos de los estudiantes. Para este caso, se aplicó la ji al cuadrado con el sexo, el semestre y si el estudiante es regular

\footnotetext{
${ }^{2}$ https://www.ibm.com/mx-es/analytics/spss-statistics-software
} 


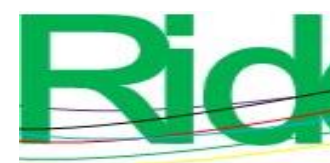

Revista lberoamericana para la Investigación y el Desarrollo Educativo

ISSN $2007-7467$

o irregular (estatus). Por tanto, se definen tres pruebas de hipótesis independientes.

- $\quad \mathrm{H}_{0 \_ \text {sexo: }}$ El sexo no influye en las opiniones de los estudiantes.

- $\quad \mathrm{H}_{1 \_ \text {sexo: }}$ El sexo sí influye en las opiniones de los estudiantes.

- $\quad \mathrm{H}_{0 \_ \text {semestre: }}$ El semestre cursado no influye en las opiniones de los estudiantes.

- $\quad \mathrm{H}_{1 \_ \text {semestre: }}$ El semestre cursado sí influye en las opiniones de los estudiantes

- $\quad \mathrm{H}_{0 \_ \text {status: }}$ El estatus no influye en las opiniones de los estudiantes.

- $\quad \mathrm{H}_{1}$ status: El estatus sí influye en las opiniones de los estudiantes.

$\mathrm{H}_{0}$ corresponde a la hipótesis nula y $\mathrm{H}_{1}$ a la hipótesis alternativa. De acuerdo con la prueba de la ji al cuadrado, si el valor de significancia es menor a 0.05 , entonces se rechaza $\mathrm{H}_{0}$ y se acepta $\mathrm{H}_{1}$, de lo contrario, se acepta $\mathrm{H}_{0}$ y se rechaza $\mathrm{H}_{1}$. Esto con un nivel de confianza de $95 \%$.

\section{Resultados}

Como se mencionó anteriormente, participaron 55 estudiantes, 43 hombres $(78.2 \%)$ y 12 mujeres (21.8 \%). Es común, al menos en la facultad de aplicación, que muy pocas mujeres se interesen en las carreras de ingenierías, por tanto, los porcentajes de mujeres son bajos respecto al de hombres.

El promedio de edad de los estudiantes fue 22.4 años (hombres $=22.4$, mujeres $=22.3$ ). Los hombres mostraron una desviación estándar mayor (2.4) al de las mujeres (1.6), lo que implica una mayor variedad de edades alejadas del promedio en los hombres.

Un total de $37(67.3 \%)$ estudiantes pertenecían al séptimo semestre y 18 (32.7\%) al noveno semestre. Además, $46(83.6 \%)$ estudiantes son considerados alumnos regulares y 9 $(16.4 \%)$ irregulares. Estos estudiantes contestaron 3.6 exámenes en pareja en promedio en la universidad.

La figura 1 muestra la preferencia de los estudiantes para resolver los exámenes en pareja. La mayoría se siente cómodo en esta forma de evaluación (90.01\%); a un bajo porcentaje le fue indiferente la forma de trabajar (9.09\%). No hubo estudiantes en desacuerdo para esta forma de evaluación. 
Figura 1. Respuestas a la afirmación "Me he sentido cómodo aplicando exámenes en pareja"

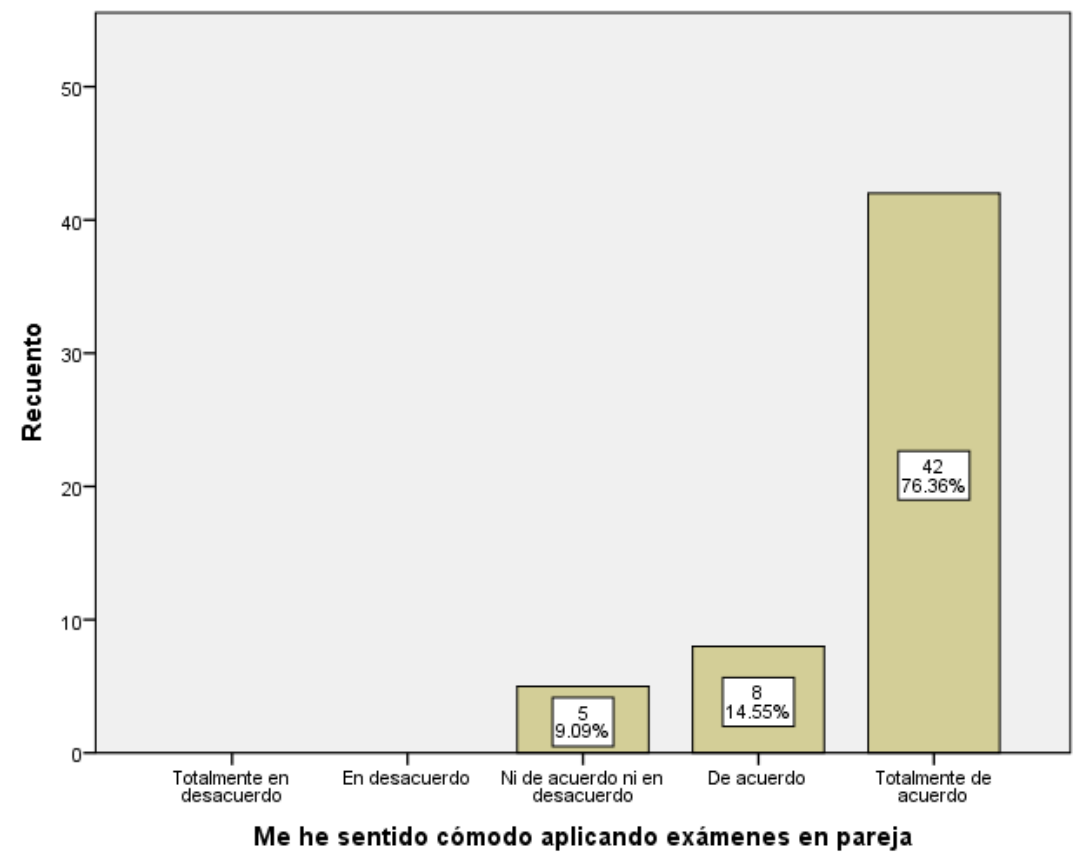

Fuente: Elaboración propia

Los estudiantes consideran que los exámenes en pareja refuerzan su conocimiento menos desarrollado: arriba de $98 \%$ está de acuerdo con la afirmación (ver figura 2).

Figura 2. Respuestas a la afirmación “Considero que los exámenes en pareja refuerzan el conocimiento que tengo débil"

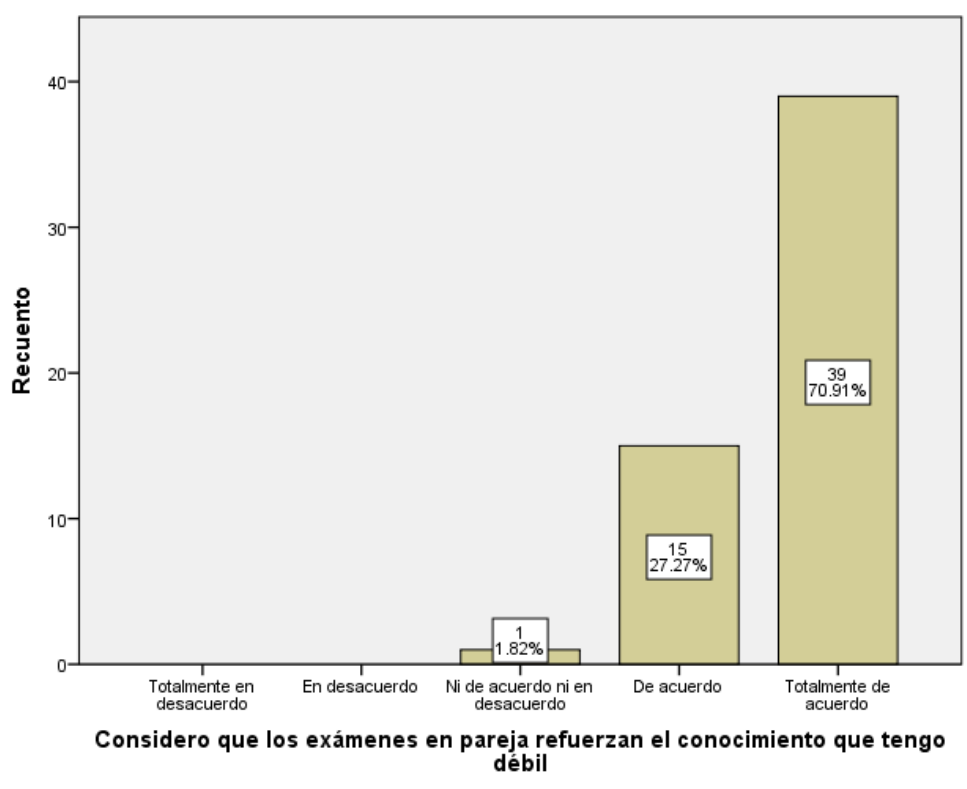

Fuente: Elaboración propia 


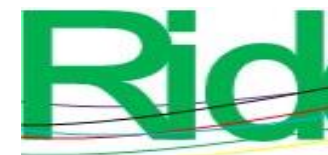

\section{Revista Iberoamericana para la Investigación y el Desarrollo Educativo ISSN 2007 - 7467}

Todos los estudiantes encuestados consideran que tienen una retroalimentación con sus compañeros al contestar exámenes en pareja. La figura 3 muestra los resultados.

La mayoría de los encuestados $(90.01 \%)$ considera que sus conocimientos son mejores al terminar los exámenes; un bajo porcentaje (9.09\%) considera neutral la afirmación (ver figura 4).

La figura 5 muestra la preferencia a los exámenes en pareja. La mayoría lo prefiere (70.91\%), aunque hay un porcentaje con indecisión (27.27 \%) y otro tanto, mínimo (1.82 \%), prefiere no hacer los exámenes en pareja.

Figura 3. Respuestas a la afirmación "Existe una retroalimentación con mis compañeros al resolver el examen en pareja"

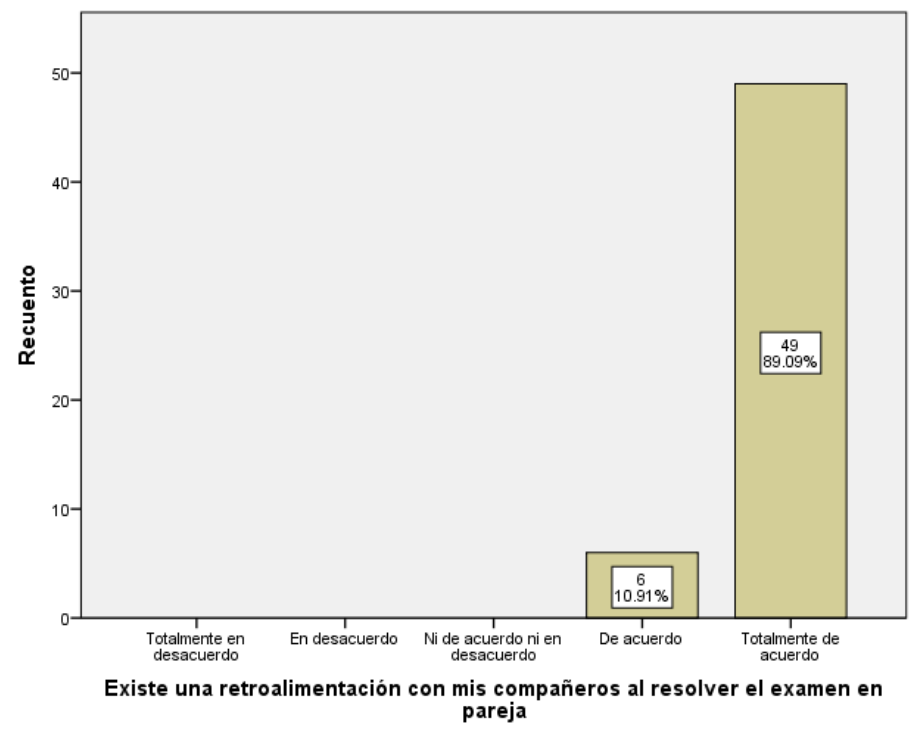

Fuente: Elaboración propia 
Figura 4. Respuestas a la afirmación "Siento que mis conocimientos son mejores al terminar

el examen en pareja que antes de aplicar el examen en pareja"

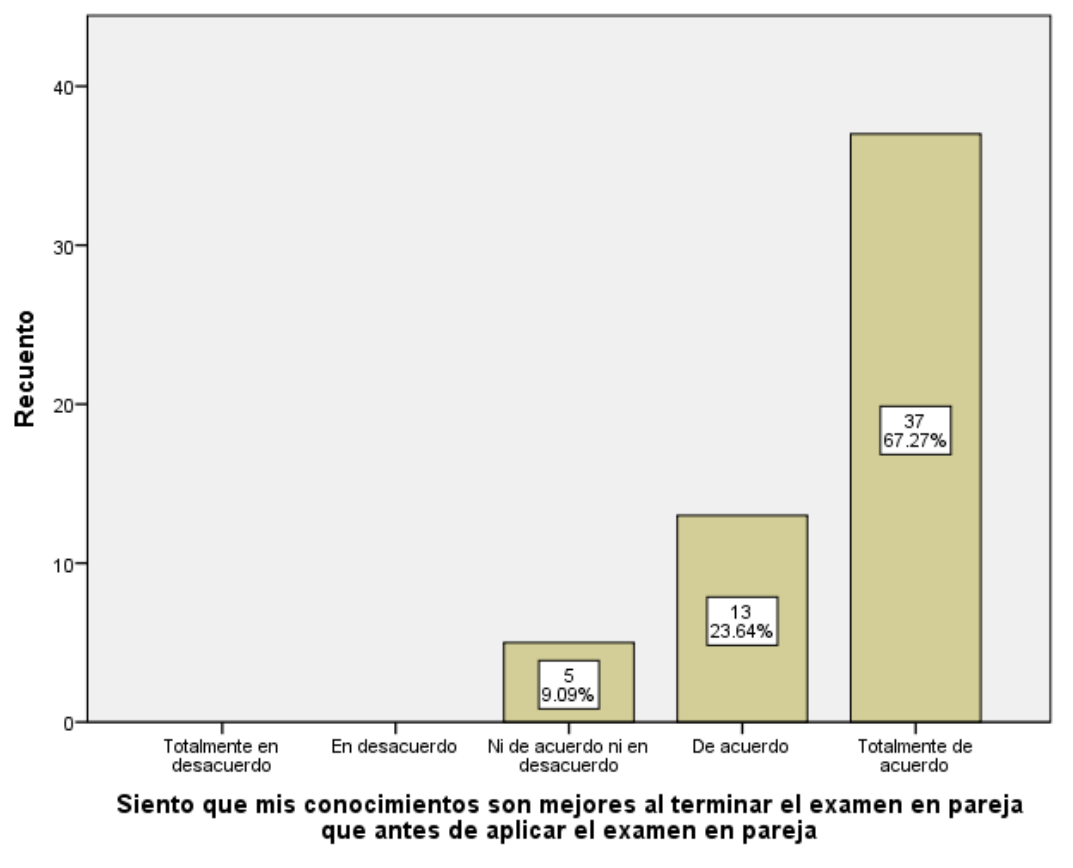

Fuente: Elaboración propia

Figura 5. Respuestas a la afirmación "Los exámenes deberían ser en pareja"

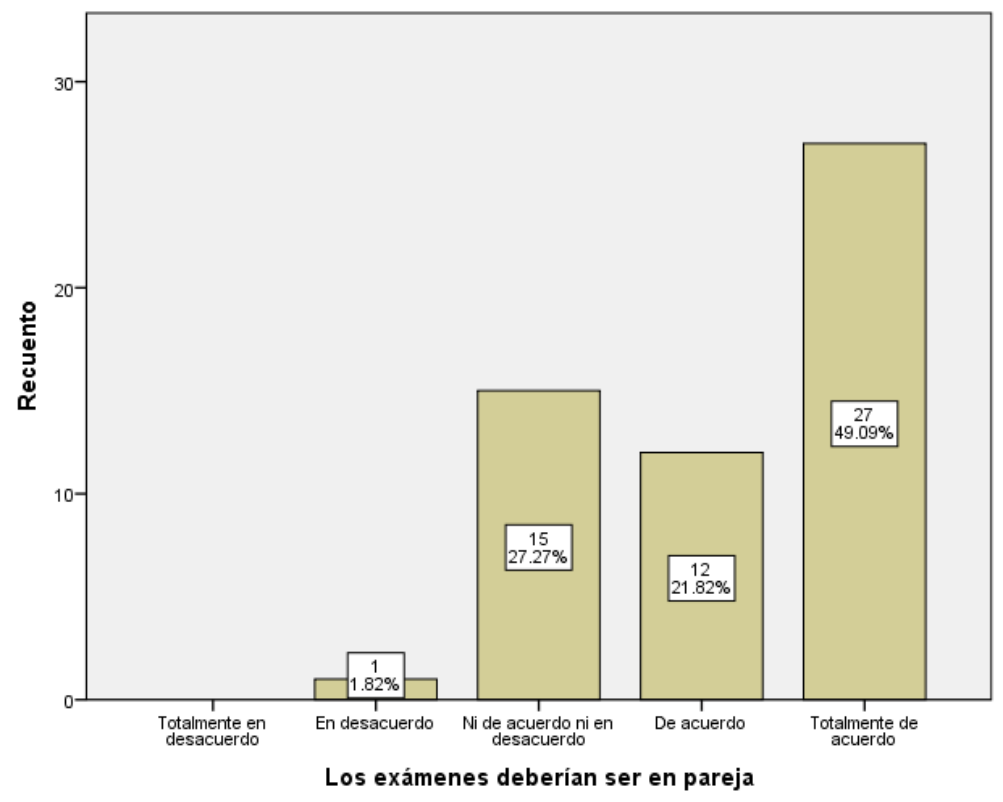

Fuente: Elaboración propia 


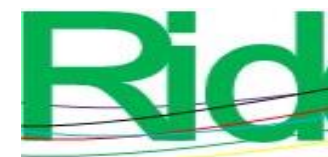

\section{Revista Iberoamericana para la Investigación y el Desarrollo Educativo ISSN 2007 - 7467}

En la última afirmación, los estudiantes consideran que los exámenes en pareja son útiles en práctica y teoría (72.7 \%), un bajo porcentaje piensa que solo en la práctica (14.5\%) y los restantes que solo en la teoría (12.7\%); ningún estudiante piensa que no son útiles este tipo de exámenes.

Ahora bien, la prueba de estaninos arrojó los datos representados en la tabla 1. Con estos valores se calcularon los puntos de corte: $a=21.3$ y $b=24.6$. Estos fueron empleados para formar las tres categorías. La tabla 2 muestra la frecuencia de las categorías definida de acuerdo con la técnica de estaninos.

Tabla 1. Estadísticos descriptivos, suma de preguntas que emplean Likert

\begin{tabular}{|c|c|c|c|c|c|}
\hline & N & Mínimo & Máximo & Media & $\begin{array}{c}\text { Desv. } \\
\text { típ. }\end{array}$ \\
\hline suma_preguntas & 55 & 17 & 25 & 23.02 & 2.198 \\
\hline N válido (según lista) & 55 & & & & \\
\hline
\end{tabular}

Fuente: Elaboración propia

Tabla 2 Frecuencias de las categorías generadas con la técnica de estaninos

\begin{tabular}{|c|c|c|c|c|c|}
\hline \multicolumn{2}{|c|}{} & Frecuencia & Porcentaje & $\begin{array}{c}\text { Porcentaj } \\
\text { e válido }\end{array}$ & $\begin{array}{c}\text { Porcentaje } \\
\text { acumulado }\end{array}$ \\
\hline Válidos & $\begin{array}{c}\text { Categoría } \\
1\end{array}$ & 12 & 21.8 & 21.8 & 21.8 \\
\cline { 2 - 6 } & $\begin{array}{c}\text { Categoría } \\
2\end{array}$ & 22 & 40.0 & 40.0 & 61.8 \\
\cline { 2 - 6 } & Categoría & 21 & 38.2 & 38.2 & 100.0 \\
& 3 & & & & \\
\hline & Total & 55 & 100.0 & 100.0 & \\
\hline
\end{tabular}

Fuente: Elaboración propia

Con los resultados presentes en la tabla 2 y las variables consideradas (sexo, semestre, estatus), se aplicó la prueba de la ji al cuadrado. El análisis estadístico indicó que no existe relación entre la opinión respecto a los exámenes cooperativos y el sexo de los estudiantes ( $p$ valor $=\mathrm{NS}, \mathrm{gl}=2$ ). Tampoco se encontró dependencia entre el semestre cursado de los estudiantes y su opinión respecto a los exámenes cooperativos en pareja $(p$-valor $=\mathrm{NS}, \mathrm{gl}=$ 

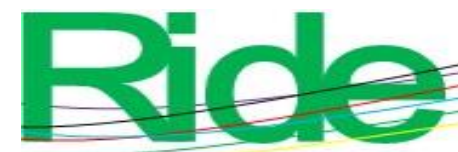

Revista Iberoamericana para la Investigación y el Desarrollo Educativo ISSN 2007 - 7467

2). Finalmente, tampoco se pudo establecer relación entre el estatus del estudiante y su opinión respecto a los exámenes cooperativos en pareja $(p$-valor $=\mathrm{NS}, \mathrm{gl}=2)$.

\section{Discusión}

La información de los resultados fue concentrada en la tabla 3. Las afirmaciones de las figuras fueron etiquetas (A1, A2 A3, A4 y A5) para poder ser referenciadas. Globalmente, se aprecia aceptación a los exámenes en pareja; solo un estudiante estuvo en desacuerdo sobre hacer los exámenes en pareja. La primera columna representa las afirmaciones y el resto es la escala de Likert. Los valores están representados en porcentajes.

En general, los alumnos se sienten cómodos con exámenes en pareja (A1), según la información recopilada. Gilley y Clarkston (2014) mencionan que los exámenes cooperativos pueden reducir la ansiedad comúnmente asociada con tomar una prueba, aumentar las relaciones positivas entre los estudiantes, mejorar la percepción del curso por parte de los estudiantes, aumentar la motivación para estudiar y disminuir la tasa de abandono de clases. Todos estos aspectos hacen que los estudiantes se sientan confiados y tranquilos respecto al examen. 


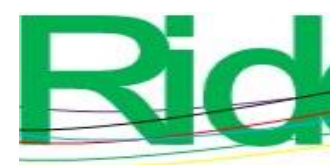

Revista Iberoamericana para la Investigación y el Desarrollo Educativo ISSN 2007 - 7467

Tabla 3. Concentrado de resultados por preguntas en escala de Likert

\begin{tabular}{|l|c|c|c|c|c|}
\hline \multicolumn{1}{|c|}{ Afirmaciones } & $\begin{array}{c}\text { Totalmen } \\
\text { te en } \\
\text { desacuer } \\
\text { do }(\%)\end{array}$ & $\begin{array}{c}\text { En } \\
\text { desacuer } \\
\text { do }(\%)\end{array}$ & $\begin{array}{c}\text { Ni de } \\
\text { acuerdo } \\
\text { ni en } \\
\text { desacuer } \\
\text { do }(\%)\end{array}$ & $\begin{array}{c}\text { De } \\
\text { acuerd } \\
\text { o }(\%)\end{array}$ & $\begin{array}{c}\text { Totalmen } \\
\text { te de } \\
\text { acuerdo } \\
(\%)\end{array}$ \\
\hline $\begin{array}{l}\text { A1. Me he sentido } \\
\text { cómodo aplicando } \\
\text { exámenes en pareja. }\end{array}$ & 0.00 & 0.00 & 9.09 & 14.55 & 76.36 \\
\hline $\begin{array}{l}\text { A2. Considero que los } \\
\text { exámenes en pareja } \\
\text { refuerzan el conocimiento } \\
\text { que tengo débil. }\end{array}$ & 0.00 & 0.00 & 1.82 & 27.27 & 70.91 \\
\hline $\begin{array}{l}\text { A3. Existe una } \\
\text { retroalimentación con mis } \\
\text { compañeros al resolver el } \\
\text { examen en pareja. }\end{array}$ & 0.00 & 0.00 & 0.00 & 10.91 & 89.09 \\
\hline $\begin{array}{l}\text { A4. Siento que mis } \\
\text { conocimientos son } \\
\text { mejores al terminar el } \\
\text { examen en pareja que } \\
\text { antes de aplicar el examen } \\
\text { en pareja. }\end{array}$ & 0.00 & 0.00 & 9.09 & 23.64 & 67.27 \\
\hline $\begin{array}{l}\text { A5. Los exámenes } \\
\text { deberían ser en pareja. }\end{array}$ & 0.00 & 1.82 & 27.27 & 21.82 & 49.09 \\
\hline
\end{tabular}

Fuente: Elaboración propia

Las afirmaciones A2, A3 y A4 están relacionadas de forma directa. Los exámenes en pareja refuerzan el conocimiento menos desarrollado de los estudiantes porque existe una retroalimentación con su pareja de examen. Esto hace que adquieran nuevos conocimientos o refuercen los conocimientos débiles al momento del examen. Los resultados muestran una aceptación hacia las tres afirmaciones con un porcentaje alto $(96.3 \%)$, solo alrededor de seis opiniones de 165 (juntando las tres afirmaciones) se consideran neutrales a las afirmaciones, aunque no en desacuerdo. Los resultados obtenidos coinciden con los de Cantwell et al. (2017), quienes argumentan que los exámenes cooperativos fomentan la participación activa en el proceso de aprendizaje a medida que los miembros del grupo discuten las preguntas del examen, el debate y la resolución de problemas para determinar las mejores respuestas y comunicar los motivos de apoyo para su respuesta particular.

Según los resultados de los estudiantes, un porcentaje alto $(70.91 \%)$ argumenta que los exámenes deberían ser en pareja. El valor es alto, pero no tanto como los porcentajes de las afirmaciones anteriores, ya que $27.27 \%$ de los estudiantes están inseguros si los exámenes 


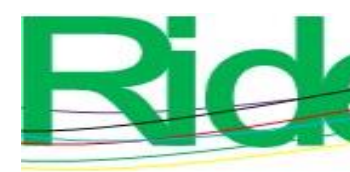

\section{Revista Iberoamericana para la Investigación y el Desarrollo Educativo ISSN 2007 - 7467}

deberían ser así. Esto implica que, a pesar de las ventajas que el método conlleva, perciben algún aspecto negativo que hace que no se inclinen por los exámenes en pareja. Los estudiantes de la investigación perciben las siguientes desventajas de los exámenes en pareja:

- $\quad$ Puede ser que el compañero de examen no tenga un nivel de conocimiento similar, lo que recargaría el trabajo cognitivo hacia un solo elemento.

- Una mentalidad cerrada de las personas para discutir adecuadamente diversas ideas u opiniones de alguna pregunta, lo que conlleva a no ponerse de acuerdo.

- $\quad$ Poca disposición al trabajo en equipo.

- Se puede dar el caso de solo dividir lo aprendido y no estudiar de forma integral, como si fuera examen individual.

- $\quad$ Aleatoriedad de compañeros al no estar disponible un estudiante preferido.

Estas desventajas coinciden con algunas de las especificadas por Mahoney y Harris (2019). Ellos argumentan que en los grupos de clase existen muchos tipos de individuos con diferentes motivaciones, personalidades y fenómenos sociales que hacen complicado controlar la colaboración de los estudiantes. Específicamente aluden a las motivaciones de los integrantes para trabajar, la compatibilidad de las personalidades de los miembros del equipo, intimidación social y conformidad, intentos por evitar situaciones embarazosas y control de los conflictos.

A pesar de algunos aspectos negativos encontrados, existen aspectos positivos. Los estudiantes de esta investigación argumentan lo siguiente:

- Existe un trabajo en equipo para complementar conocimientos entre compañeros.

- $\quad$ Existe un diálogo y razonamiento para reforzar el conocimiento.

- $\quad$ Se pueden complementar aspectos teóricos y prácticos.

- $\quad$ Reduce el estrés impuesto por la evaluación.

- Genera confianza para contestar el examen.

Estos aspectos aparecen replicados en Leight, Saunders, Calkins, y Withers (2012) y Cantwell et al. (2017). Ambos grupos de investigadores mencionan que los estudiantes perciben que aprenden mejor en los exámenes cooperativos y exhiben mejores puntajes que en las pruebas individuales. Además, mejoran las habilidades de pensamiento crítico, mejoran la colaboración y el trabajo en equipo entre pares, incrementan las habilidades de aprendizaje y la motivación para estudiar material, reducen la ansiedad por los exámenes y, en general, 


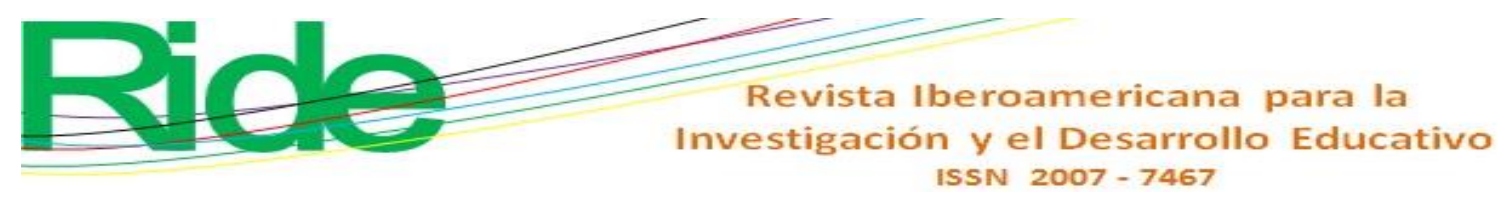

logran un mejor desempeño y una mayor confianza a la hora de ser evaluados.

Un aspecto importante en esta investigación es el número de personas en el equipo. $\mathrm{Al}$ ser solo dos elementos, se requiere de un trabajo en equipo real, para complementar las respuestas de ambos y generar retroalimentación. Por el contrario, más de dos estudiantes en el equipo podría traer un desequilibrio. Alarcón et al. (2018) refuerzan esta idea al precisar que, si bien un gran número de actividades pueden realizarse en grupos, en muchas ocasiones uno o dos estudiantes terminan por hacer el trabajo de todo el equipo. Si reducimos al mínimo el equipo de trabajo, tendremos más probabilidad de una correcta evaluación, sin dar calificación a estudiantes que no aportan al equipo. Por tanto, esta investigación considera que dos elementos son los indicados para los exámenes cooperativos, por lo que se eligió aplicar exámenes en pareja.

Los estudiantes opinan que este tipo de exámenes puede ser aplicado tanto en teoría como en práctica. De acuerdo con Pressman (2010), la ingeniería de software está formada por procesos, métodos o prácticas y herramientas que permiten a los profesionales desarrollar software informático de alta calidad. Por tanto, aspectos teóricos tienen que ser analizados antes de entrar en la práctica, por lo que es común aplicar exámenes teóricos y prácticos en la carrera, incluso al mismo tiempo en algunos casos.

Por último, el análisis estadístico para las tres pruebas de hipótesis elaboradas determina que los valores de significancia ( $\operatorname{sexo}=0.396$, semestre $=0.449$ y estatus $=0.153$ ) no son menores a 0.05 , por tanto, se acepta la hipótesis nula para cada caso. Esto indica que el sexo, el semestre y el estatus no tienen relación con las opiniones de los estudiantes; es decir, estas variables se comportan de forma indiferente ante los exámenes cooperativos. Es probable que otras variables como la personalidad, la percepción del estudiante respecto a su pareja, afinidad entre parejas, la materia en evaluación, por mencionar algunas, puedan influir en la percepción del alumno con respecto a esta metodología de evaluación, así como con respecto a las calificaciones finales o parciales bajo esta metodología.

Sin embargo, aún con todas estas bondades del trabajo cooperativo, en las escuelas todavía persiste la idea de que el trabajo en grupos pequeños puede ser una pérdida de tiempo que no garantiza el éxito en el rendimiento académico de los estudiantes y prefieren continuar con el modelo tradicional. La justificación es errónea. Lara (2005) establece que eso pasa porque se mira al alumno como un mero depositario de información que hay que asignarle un número para calificar sus habilidades de tipo académico. Igualmente, subyace la idea de que 


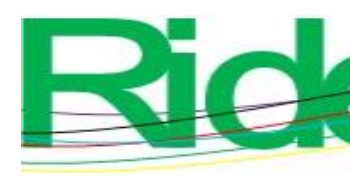

Revista Iberoamericana para la Investigación y el Desarrollo Educativo ISSN 2007 - 7467

todos son iguales y aprenderán de la misma manera. Además, como hay que evaluar conocimientos aprendidos de memoria sobre pruebas de papel y lápiz, deben imperar actividades que fomenten el individualismo y la memorización. Este modelo tradicional está frenando el desarrollo de habilidades sociales, tal y como el trabajo en equipo, la toma de decisiones, el liderazgo, la organización y la proactividad. Muchas de estas cualidades comienzan a ser cada vez más demandadas en el ámbito profesional, en específico y más recientemente en las áreas de computación, y, por tanto, los alumnos necesitan desarrollarlas a lo largo de su formación.

\section{Conclusiones}

Esta investigación diseñó un instrumento para recopilar información con el objetivo de determinar si los estudiantes de ingeniería de Software de la Facultad de Ingeniería Mochis de la Universidad Autónoma de Sinaloa tienden a aceptar los exámenes en pareja. Para ello, se aplicaron exámenes cooperativos en pareja durante un semestre. Además, se diseñó un instrumento para recopilar la opinión de los estudiantes.

Los resultados indican una aceptación de los exámenes cooperativos en pareja. Se puede argumentar que el aprendizaje ocurre a través de los exámenes cooperativos porque los estudiantes tienen la oportunidad de desarrollar, reconstruir y avanzar activamente su conocimiento a través de la interacción con otros. Los resultados también indican que la aceptación de los exámenes cooperativos no está relacionada con el sexo, estatus o el semestre.

Los participantes señalan que este método tiene aspectos negativos que afectan la colaboración entre compañeros, en especial si la pareja no cuenta con conocimientos regulares para presentar el examen. Por otra parte, los estudiantes indican aspectos positivos, de retroalimentación con sus compañeros y aumento de confianza para presentar el examen, principalmente. Los proyectos en conjunto, si bien son estrategias de aprendizaje centradas en la reflexión, análisis y comprensión de los contenidos, también provocan el desarrollo de competencias en la resolución de problemas, liderazgo y contribuyen a forjar un espíritu capaz de crear ambientes en el que el debate proporciona un nivel desarrollo del pensamiento más organizado y sistemático. Sin duda, en la interacción con otros, se dan oportunidades donde los estudiantes estimulan sus zonas de desarrollo próximo. 


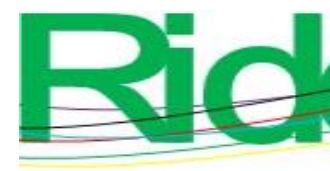

\section{Revista Iberoamericana para la Investigación y el Desarrollo Educativo ISSN 2007 - 7467}

Como trabajo futuro, se planea analizar la aceptación de las técnicas de trabajo cooperativo en áreas de desarrollo de software. Por otra parte, el estudio tiene un enfoque hacia el estudiante, aunque también puede ser analizada la perspectiva del profesor: una línea de investigación más. Además, se podría establecer otra investigación enfocada en comprobar que el conocimiento adquirido a través de los exámenes cooperativos es un conocimiento más sustancial que el adquirido a través de los exámenes individuales. El trabajo cooperativo se consolida cada vez más como una estrategia didáctica válida y pertinente que indudablemente proporciona beneficios en la formación profesional. Ahora más que nunca se requiere del manejo de herramientas sociales e interculturales y el desarrollo de habilidades sociales y de cooperación por parte de los ingenieros de software.

\section{Referencias}

Alarcón, E., Sepúlveda, M. P. y Madrid, D. (2018). Qué es y qué no es aprendizaje cooperativo. Ensayos: Revista de la Facultad de Educación de Albacete, 33(1), 205-220. Recuperado de https://dialnet.unirioja.es/servlet/articulo?codigo=6536516.

Amores, E. (2016). El aprendizaje cooperativo a examen: Resultados de una experiencia longitudinal. (tesis de pregrado). Universidad de Castilla-La Mancha, España.

Cantwell, E. R., Sousou, J., Jadotte, Y. T., Pierce, J. and Akioyamen, L. E. (2017). Protocol: Collaborative Testing for Improving Student Learning Outcomes and Test-Taking Performance in Higher Education: A Systematic Review. Campbell Systematic $\begin{array}{llll}\text { Reviews, } & 13(1), 18 . & \text { Retrieved } & \text { from }\end{array}$ https://onlinelibrary.wiley.com/doi/full/10.1002/CL2.186.

Centrella, A. (2012). Collaborative testing as posttest review. Nursing Education Perspectives, 33(1), 340-341.

Díaz, F. y Hernández, G. (2010). Estrategias docentes para un aprendizaje significativo: Una interpretación constructivista (3.a ed.). México: McGraw-Hill Education.

Gilley, B. and Clarkston, B. (2014). Research and Teaching: Collaborative Testing: Evidence of Learning in a Controlled In-Class Study of Undergraduate Students. Journal of College Science Teaching, 43(3), 83-91. Retrieved from https://doi.org/10.2505/4/jcst14_043_03_83.

Hernández, R., Fernández, C. y Baptista, M. (2014). Metodología de la investigación (6.a ed.). México: McGraw-Hill. 


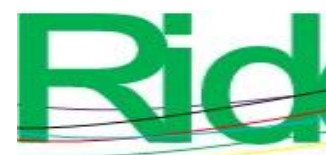

Revista Iberoamericana para la Investigación y el Desarrollo Educativo

ISSN 2007 - 7467

Johnson, D. and Johnson, R. (2004). Assessing Students in Groups: Promoting Group Responsibility and Individual Accountability. United States: Corwin Press.

Lara, R. S. (2005). El aprendizaje cooperativo: un modelo de intervención para los programas de tutoría escolar en el nivel superior. Revista de la Educación Superior, 34(133), 87104.

Leight, H., Saunders, C., Calkins, R. and Withers, M. (2012). Collaborative testing improves performance but not content retention in a large-enrollment introductory biology class. CBE- Life Sciences Education, 11(4), 392-401.

Mahoney, J. W. and Harris, B. (2019). The effects of collaborative testing on higher order thinking: Do the bright get brighter? Active Learning in Higher Education, 20(1), 25 37. Retrieved from https://doi.org/10.1177/1469787417723243.

Núñez, J. (2017). Los métodos mixtos en la investigación en educación: Hacia un uso reflexivo. Cadernos de Pesquisa, 47(164), 632-649. Recuperado de https://doi.org/10.1590/198053143763.

Pressman, R. (2010). Software Engineering: A Practitioner's Approach (7th ed.). New York, United States: McGraw-Hill.

Revelo, O., Collazos, C. and Jiménez, J. (2018). Collaborative work as a didactic strategy for teaching/learning programming: a systematic literature review programming. TecnoLógicas, 21(41), 115-134.

Thorndike, R. L. (1982). Applied Psychometrics. Houghton Mifflin College Division. 


\begin{tabular}{|c|c|}
\hline Rol de Contribución & Autor (es) \\
\hline Conceptualización & Alan Ramírez-Noriega \\
\hline Metodología & Alan Ramírez-Noriega \\
\hline Software & No aplica \\
\hline Validación & Samantha Jiménez \\
\hline Análisis Formal & $\begin{array}{l}\text { Samantha Jiménez (Principal) } \\
\text { Alan Ramírez-Noriega (Apoyo) }\end{array}$ \\
\hline Investigación & Yobani Martínez-Ramírez \\
\hline Recursos & Yobani Martínez-Ramírez \\
\hline Curación de datos & Samantha Jiménez \\
\hline $\begin{array}{l}\text { Escritura - Preparación del } \\
\text { borrador original }\end{array}$ & $\begin{array}{l}\text { José Emilio Sánchez García (Principal) } \\
\text { Alan Ramírez-Noriega (Apoyo) }\end{array}$ \\
\hline $\begin{array}{l}\text { Escritura - Revisión y } \\
\text { edición }\end{array}$ & $\begin{array}{l}\text { José Emilio Sánchez García (Principal) } \\
\text { Alan Ramírez-Noriega (Apoyo) } \\
\text { Yobani Martínez-Ramírez (Apoyo) }\end{array}$ \\
\hline Visualización & José Emilio Sánchez García \\
\hline Supervisión & Alan Ramírez-Noriega \\
\hline $\begin{array}{l}\text { Administración de } \\
\text { Proyectos }\end{array}$ & Alan Ramírez-Noriega \\
\hline Adquisición de fondos & $\begin{array}{l}\text { Alan Ramírez-Noriega } \\
\text { Samantha Jiménez } \\
\text { Yobani Martínez-Ramírez } \\
\text { José Emilio Sánchez García } \\
\text { Contribución igual }\end{array}$ \\
\hline
\end{tabular}

